\title{
Changes in Tillering, Nutritional Status, and Biomass Yield of Panicum Maximum Used for Cadmium Phytoextraction
}

\section{Flávio Henrique Silveira Rabêlo ( $\boldsymbol{\nabla}$ flaviohsr.agro@usp.br)}

Universidade de São Paulo - Câmpus Luiz de Queiroz: Universidade de Sao Paulo Escola Superior de Agricultura Luiz de Queiroz https://orcid.org/0000-0002-8804-7182

\section{Felipe dos Santos}

Universidade de São Paulo - Câmpus Luiz de Queiroz: Universidade de Sao Paulo Escola Superior de Agricultura Luiz de Queiroz

\section{José Lavres}

Universidade de São Paulo Centro de Energia Nuclear na Agricultura: Universidade de Sao Paulo Centro de Energia Nuclear na Agricultura

\section{Luís Alleoni}

Universidade de São Paulo - Câmpus Luiz de Queiroz: Universidade de Sao Paulo Escola Superior de Agricultura Luiz de Queiroz

\section{Research Article}

Keywords:

Posted Date: January 14th, 2022

DOI: https://doi.org/10.21203/rs.3.rs-1247155/v1

License: (c) (1) This work is licensed under a Creative Commons Attribution 4.0 International License. Read Full License 


\section{Abstract}

Although several grasses have been evaluated for cadmium (Cd) phytoextraction, there are no studies assessing how $\mathrm{Cd}$ is accumulated and distributed in the tissues of Panicum maximum grown in mildly polluted soils. The evaluation of tillering, nutritional status and biomass yield of this grass, mainly along successive shoot regrowths, is not well studied so far. Thus, $P$. maximum Jacq. cv. Massai was grown for two periods in an Oxisol presenting bioavailable Cd concentrations varying from 0.04 (control) to $10.91 \mathrm{mg}$ $\mathrm{kg}^{-1}$ soil. Biomass yield of leaves and stems' growth have decreased under the highest $\mathrm{Cd}$ exposure, but it did not occur in the regrowth period, indicating that Cd-induced toxicity is stronger in the early stages of development of $P$. maximum. The tillering was not compromised even the basal node presenting $\mathrm{Cd}$ concentrations higher than $100 \mathrm{mg} \mathrm{kg}^{-1} \mathrm{DW}$. We identified a restriction on $\mathrm{Cd}$ transport upwards from basal node, which was the main local of $\mathrm{Cd}$ accumulation. Apparently, $\mathrm{P}, \mathrm{K}, \mathrm{Mg}, \mathrm{S}$ and $\mathrm{Cu}$ are involved in processes that restrict $\mathrm{Cd}$ translocation and confer high tolerance to $\mathrm{Cd}$ in $P$. maximum. The $\mathrm{Cd}$-induced nutritional disorders did not negatively correlate with factors used to calculate phytoextraction efficiency. However, the nutritional adjustments of $P$. maximum to cope with $\mathrm{Cd}$ stress restricted the upward $\mathrm{Cd}$ transport, which decreased the phytoextraction efficiency from the available Cd concentration of $5.93 \mathrm{mg} \mathrm{kg}^{-1}$ soil.

\section{Introduction}

Several species of grass have been evaluated for cadmium (Cd) phytoextraction as they present desirable characteristics such as high biomass yield, fast growth, and successive shoot regrowth after shoot harvest (Rabêlo et al. 2021a). Between the grasses assessed, Panicum maximum has stood out for surviving when exposed to very high Cd concentrations (de Anicésio and Monteiro 2021), despite Cd is more transported to aboveground plant tissues at such conditions, compromising tillers emission, inducing nutritional disorders, and decreasing biomass yield, which results in lowered Cd phytoextraction efficiency (de Anicésio and Monteiro 2019). As tillering and nutrient homeostasis are essential for grass development, the use of $P$. maximum for $\mathrm{Cd}$ phytoextraction is supposed to be feasible only in mildly polluted soils (Rabêlo et al. 2021a), otherwise, the Cd-induced phytotoxicity could prevent further growth and biomass yield. However, to the best of our knowledge, there are no studies reporting how $\mathrm{Cd}$ is accumulated and distributed into the tissues of $P$. maximum in mildly polluted soils and how it affects the tillering, nutritional status and biomass yield of this grass, especially along successive shoot regrowth.

Each tiller in grasses is originated from the emission of a single apical meristem initiated in the axillary buds located at the basal node of the plant (Chrysler 1906). In other species of the family Poaceae, such as rice (Oryza sativa), there is evidence that $\mathrm{Cd}$ is highly accumulated in the basal node of such plant and compromises the tillering (Fujimaki et al., 2010). Nutritional disorders also can decrease the tillering of $P$. maximum by increasing the number of dormant buds (Garcez Neto et al. 2012). As $\mathrm{Cd}$ strongly induces nutritional disorders at high concentrations in such grass (Rabêlo et al. 2020a), the relationship between Cd accumulation, tillering and biomass yield of $P$. maximum used for $\mathrm{Cd}$ phytoextraction in mildly polluted soil should have better understood. Thereby, our aims with this study were to i) assess how $\mathrm{Cd}$ affects the growth and biomass yield of $P$. maximum used for $\mathrm{Cd}$ phytoextraction in mildly polluted soil along two successive shoot growths; ii) identify the main local of $\mathrm{Cd}$ accumulation (basal node?) in P. maximum used 
for Cd phytoextraction; iii) check if a possible $\mathrm{Cd}$ accumulation in the basal node could limit successive shoot emissions due to reduced tillering; and iv) diagnose eventual nutritional disorders Cd-induced and correlate such event with the Cd phytoextraction efficiency of $P$. maximum.

\section{Materials And Methods}

\section{Soil collection and physic-chemical characterization}

The soil was collected from the upper layer (0.0-0.2 $\mathrm{m}$ depth) of an area under native pasture in Piracicaba, state of São Paulo, Brazil (S 22 $43^{\prime} 04^{\prime \prime} ;$ W 47 $\left.36^{\circ} 55^{\prime \prime}\right)$. The soil was classified as Typic Hapludox (USDA 1999). Soil characteristics were determined on air-dried soil sieved with a 2-mm (Table 1). The determination of $\mathrm{pH}\left(0.01 \mathrm{~mol} \mathrm{~L}^{-1} \mathrm{CaCl}_{2}\right)$, Al (extraction with $1 \mathrm{~mol} \mathrm{~L}^{-1} \mathrm{KCl}$ ) and soil organic matter (oxidation with $0.2 \mathrm{~mol}$ $\mathrm{L}^{-1} \mathrm{~K}_{2} \mathrm{Cr}_{2} \mathrm{O}_{7}$ ) followed the methods of the Brazilian Agricultural Research Corporation - EMBRAPA (EMBRAPA 1997). Potential acidity (H+Al) was estimated following the method of pH SMP (Raij et al. 2001). The available concentrations of $\mathrm{P}, \mathrm{K}, \mathrm{Ca}$ and $\mathrm{Mg}$ were extracted with ion exchange resin (Raij et al. 2001), S with $0.01 \mathrm{~mol} \mathrm{~L}^{-1} \mathrm{Ca}\left(\mathrm{H}_{2} \mathrm{PO}_{4}\right)_{2}$ (Tabatabai and Bremner 1970), B with hot water in a microwave oven (Bataglia and Raij 1990), and $\mathrm{Cu}, \mathrm{Fe}, \mathrm{Mn}$ and $\mathrm{Zn}$ with diethylene triamine pentaacetic acid - DTPA at pH 7.3 (Abreu et al. 2001). From these results the sum of bases, effective cation exchange capacity, total cation exchange capacity, base saturation, and saturation by aluminum were calculated (EMBRAPA 1997). The initial pseudo-total Cd concentration was determined following the method 3050B proposed by the United States Environmental Protection Agency - USEPA (USEPA 1996). The granulometric fractions sand, silt and clay were obtained by the hydrometer method (Gee and Bauder 2002). 
Table 1

Descriptive analysis of chemical and physical properties of the Typic Hapludox used in this study

\begin{tabular}{|c|c|c|c|}
\hline $\mathrm{pH} 0.01 \mathrm{~mol} \mathrm{~L}^{-1} \mathrm{CaCl}_{2}$ & 4.6 & $\mathrm{Al}\left(\mathrm{mmol}_{\mathrm{c}} \mathrm{dm}^{-3}\right)$ & 2 \\
\hline & & $\mathrm{H}+\mathrm{Al}\left(\mathrm{mmol}_{\mathrm{c}} \mathrm{dm}^{-3}\right)$ & 25 \\
\hline $\mathrm{P}\left(\mathrm{mg} \mathrm{dm}^{-3}\right)$ & 4 & & \\
\hline $\mathrm{S}\left(\mathrm{mg} \mathrm{dm}^{-3}\right)$ & 4.5 & $\mathrm{SB}\left(\mathrm{mmol}_{\mathrm{C}} \mathrm{dm}^{-3}\right)$ & 15 \\
\hline $\mathrm{K}\left(\mathrm{mmol}_{\mathrm{C}} \mathrm{dm}^{-3}\right)$ & 0.5 & $\mathrm{CEC}_{\mathrm{t}}\left(\mathrm{mmol}_{\mathrm{C}} \mathrm{dm}^{-3}\right)$ & 40 \\
\hline $\mathrm{Ca}\left(\mathrm{mmol}_{\mathrm{C}} \mathrm{dm}^{-3}\right)$ & 10.5 & $\mathrm{CEC}_{\mathrm{e}}\left(\mathrm{mmol}_{\mathrm{c}} \mathrm{dm}^{-3}\right)$ & 17 \\
\hline $\mathrm{Mg}\left(\mathrm{mmol}_{\mathrm{C}} \mathrm{dm}^{-3}\right)$ & 4 & & \\
\hline $\mathrm{B}\left(\mathrm{mg} \mathrm{dm}^{-3}\right)$ & 0.2 & BS $(\%)$ & 37 \\
\hline $\mathrm{Cu}\left(\mathrm{mg} \mathrm{dm}^{-3}\right)$ & 0.5 & m (\%) & 11 \\
\hline $\mathrm{Fe}\left(\mathrm{mg} \mathrm{dm}^{-3}\right)$ & 39 & & \\
\hline $\operatorname{Mn}\left(\mathrm{mg} \mathrm{dm}^{-3}\right)$ & 6.4 & $\operatorname{SOM}\left(\mathrm{g} \mathrm{kg}^{-1}\right)$ & 14 \\
\hline \multirow[t]{2}{*}{$\mathrm{Zn}\left(\mathrm{mg} \mathrm{dm}^{-3}\right)$} & 2.1 & Sand $\left(\mathrm{g} \mathrm{kg}^{-1}\right)$ & 828 \\
\hline & & Silt $\left(\mathrm{g} \mathrm{kg}^{-1}\right)$ & 23 \\
\hline $\mathrm{Cd}\left(\mathrm{mg} \mathrm{kg}^{-1}\right)$ & 0.67 & Clay $\left(\mathrm{g} \mathrm{kg}^{-1}\right)$ & 149 \\
\hline
\end{tabular}

\section{Plant Material And Experimental Set-up}

Panicum maximum Jacq. Cv. Massai was grown under greenhouse under natural conditions $\left(31.5 \pm 5^{\circ} \mathrm{C}\right.$ and $63.7 \pm 14 \%$ relative humidity during plant growth, and $26.0 \pm 5^{\circ} \mathrm{C}$ and $66.0 \pm 12 \%$ relative humidity during plant regrowth), in pots containing $5 \mathrm{~kg}$ of the Typic Hapludox (Table 1). Treatments were composed by a control ( $0.67 \mathrm{mg} \mathrm{Cd} \mathrm{kg}^{-1}$ soil, Table 1$)$ and three added $\mathrm{Cd}$ doses attempting to reach the final pseudototal Cd concentrations of 7.2, 14.4 and $28.8 \mathrm{mg} \mathrm{kg}^{-1}$ soil. Such $\mathrm{Cd}$ concentrations were defined from the study of Farnezi et al. (2020), in which the authors estimated that $P$. maximum can grow up in soils presenting $\mathrm{Cd}$ concentrations close to $30 \mathrm{mg} \mathrm{kg}^{-1}$ soil. However, the final $\mathrm{Cd}$ concentrations reached were lower (Fig. 1). Pots were distributed in completely randomized design with four replicates per condition. 


\section{Soil Pollution, Growth Conditions And Plant Harvesting}

The Oxisol was spiked with $\mathrm{Cd}$ by using $\mathrm{CdCl}_{2}$, and then incubated for 30 days. The soil water content was maintained at constant level ( $70 \%$ of the maximum water holding capacity) throughout the study by adding deionized water. The basic fertilization was performed after the soil incubation by applying $100 \mathrm{mg} \mathrm{N}$ $\left(\mathrm{NH}_{4} \mathrm{NO}_{3}\right), 150 \mathrm{mg} \mathrm{P}\left(\mathrm{KH}_{2} \mathrm{PO}_{4}\right), 100 \mathrm{mg} \mathrm{K}\left(\mathrm{KH}_{2} \mathrm{PO}_{4}\right)$ and $50 \mathrm{mg} \mathrm{S}\left(\mathrm{MgSO}_{4} .7 \mathrm{H}_{2} \mathrm{O}\right)$ per $\mathrm{kg}$ of soil, according to Werner et al. (1997). Seeds of $P$. maximum Jacq. cv. Massai were sown in the same day of the basic fertilization. A thinning leaving 10 seedlings per pot was carried out 21 days after sowing. Twenty-six days after sowing, the fertilization with $100 \mathrm{mg} \mathrm{N}\left(\mathrm{NH}_{4} \mathrm{NO}_{3}\right)$ and $100 \mathrm{mg} \mathrm{K}(\mathrm{KCl})$ per $\mathrm{kg}$ of soil was performed on the top (Werner et al. 1997). Aboveground plant parts were harvested $(5 \mathrm{~cm}$ above the basal node to allow plant regrowth; Pautler et al. 2013) 54 days after sowing, adopting the beginning of the senescence of the mature leaves of plants in a more advanced physiological stage as the criterion for the harvest (Rabêlo et al. 2017).

To stimulate shoot regrowth, $100 \mathrm{mg} \mathrm{N}\left(\mathrm{NH}_{4} \mathrm{NO}_{3}\right)$ and $100 \mathrm{mg} \mathrm{K}(\mathrm{KCl})$ per $\mathrm{kg}$ of soil were top dressed one day after the first shoot harvest (Werner et al. 1997). Twenty-five days after, a second top-dressed fertilization was performed with $100 \mathrm{mg} \mathrm{N}\left(\mathrm{NH}_{4} \mathrm{NO}_{3}\right)$ and $100 \mathrm{mg} \mathrm{K} \mathrm{(KCl)}$ per $\mathrm{kg}$ of soil (Werner et al. 1997). Nutrients were applied through a solution in the first and second growths. The second and final harvest was made 59 days after the first harvest, adopting the same criterion for the first harvest.

At the end of the study, the plant material was separated into roots, basal node, and shoot. The shoot collected at the end of the two growth periods was divided from the top to basal node into leaves I (the first fully expanded leaf), II, III and other leaves (leaves IV, V etc.), and stems. We counted the number of tillers one day before the first and second harvests. After the end of both growth periods the fresh weight of each plant tissue was recorded. Then, the plant tissues were placed in a forced ventilation oven at $60^{\circ} \mathrm{C}$ for $72 \mathrm{~h}$ to determine the dry weight and the concentrations of nutrients and $\mathrm{Cd}$.

\section{Determination of the concentration of nutrients and $\mathrm{Cd}$ in the plant tissues}

The dried material was ground in a Wiley type mill (Model 4, Thomas Scientific, Swedesboro, NJ, USA). For determination of the concentrations of $\mathrm{P}, \mathrm{K}, \mathrm{Ca}, \mathrm{Mg}, \mathrm{S}, \mathrm{B}, \mathrm{Cu}, \mathrm{Fe}, \mathrm{Mn}, \mathrm{Zn}$ and $\mathrm{Cd}$, the plant material was digested in a microwave oven (Model ultraWAVE SRC - Single Reaction Chamber - Technology, Milestone, Sorisole, Italy) by using a mixture composed by nitric acid $\left(\mathrm{HNO}_{3} 20 \%\right)$ and hydrogen peroxide $\left(\mathrm{H}_{2} \mathrm{O}_{2} 30 \%\right)$ $(\mathrm{v} / \mathrm{v})$, according to USEPA 3051A method (USEPA 2007). The contents were determined by inductively coupled plasma optical emission spectrometry (ICP-OES, iCAP 7000 SERIES, Thermo Fisher Scientific, Waltham, USA). Standard reference material (SRM 1515 - apple leaves) and blank reagent samples were used during the digestion to assure the accuracy and precision of the extraction and analytical method.

\section{Calculation Of Nutrient Use Efficiency (Nue)}


The NUE ( $\mathrm{g}^{2} \mathrm{mg}^{-1}$ for macronutrients and $\mathrm{g}^{2} \mathrm{ng}^{-1}$ for micronutrients) was calculated for each nutrient: NUE $=\left[(\text { dried biomass of the plant tissue })^{2} /\right.$ nutrient accumulated in the plant tissue $]$ (Siddiqi and Glass 1981), where nutrient accumulated in the plant tissue (mg/plant for macronutrients and $\mu \mathrm{g} / \mathrm{plant}$ for micronutrients) was obtained by multiplying the nutrient concentration ( $\mathrm{g} \mathrm{kg}^{-1} \mathrm{DW}$ for macronutrients and $\mathrm{mg} \mathrm{kg}^{-1} \mathrm{DW}$ for micronutrients) in each tissue by the dry weight of the respective tissue.

\section{Calculation Of The Factors Related To Cd Phytoextraction Efficiency}

Cadmium phytoextraction efficiency was calculated through the bioconcentration factor - BCF $(\mathrm{BCF}=\mathrm{Cd}$ concentration in the aerial tissue / $\mathrm{Cd}$ concentration extracted by $\mathrm{CaCl}_{2}$ in the Oxisol) and translocation factor - TF (TF = sum of Cd accumulated in the aerial tissues in the two growth periods / Cd accumulated in the roots) (adapted from Ali et al. 2013).

\section{Statistical analysis}

Normality and homoscedasticity were checked before proceeding with the analysis of variance. Then, the data were submitted to analysis of variance and post-hoc Tukey test $(p<0.05)$ to compare the means between $\mathrm{Cd}$ concentrations within each plant tissue for each growth period. Two-by-two comparisons were conducted using the t-test to compare the means between growth periods within each Cd concentration for each plant tissue. The statistical analyses were performed in the Statistical Analysis System v. 9.2 (SAS Institute 2008), whereas the graphs were plotted with SigmaPlot v. 10.0 (Systat Software Inc., Chicago, Illinois, USA). Results were expressed as mean \pm standard error of the mean (SEM).

\section{Results}

The highest $\mathrm{Cd}$ concentrations decreased shoot biomass yield of P. maximum in the growth but not in the regrowth period, indicating that $\mathrm{Cd}$ is more harmful in the early stages of development

Only the leaf and stem biomass collected at the end of the growth period of $P$. maximum grown under the available Cd concentration of $10.91 \mathrm{mg} \mathrm{kg}^{-1}$ soil was lower compared to control (Figs. 2A-B). The leaf and stem biomass of $P$. maximum at the end of the regrowth period was not affected by Cd concentrations in the Oxisol, as well as the biomass of basal node and roots (Figs. 2A-D). Plants of the control treatment presented lower leaf biomass in the regrowth compared to growth period of $P$. maximum, but when this grass was exposed to the available Cd concentration of $10.91 \mathrm{mg} \mathrm{kg}^{-1}$ soil the opposite was observed (Fig. 2A). The stem biomass of P. maximum was higher in the regrowth compared to growth period, regardless of $\mathrm{Cd}$ concentration in the Oxisol (Fig. 2B), which indicates that other factor than the own $\mathrm{Cd}$ concentration affected stem biomass yield of this grass.

The number of tillers emitted by $P$. maximum was not affected by the available $\mathrm{Cd}$ concentrations in the Oxisol, in both growth periods. However, except for the control treatment, the number of tillers emitted during 
regrowth period was higher compared to the growth period (Fig. 3A). Cadmium accumulated in the basal node presented a trend to reduce the number of tillers of $P$. maximum only in the growth period (Fig. 3B). Conversely from which was observed for the number of tillers, the leaf/stem ratio of $P$. maximum exposed to the higher available $\mathrm{Cd}$ concentration increased in relation to control (Fig. 3C) due to the more pronounced Cd-induced inhibition on the stem than leaf biomass yield under such conditions (Figs. 2A-B). The leaf/stem ratio in the regrowth period of $P$. maximum was not affected by the available $\mathrm{Cd}$ concentrations. Regardless of Cd concentration in the Oxisol, the leaf/stem ratio was lower in the regrowth compared to growth period (Fig. 3C), which reinforce the assumption that other factor than the own Cd concentration affected the grass regrowth.

\section{Cadmium concentration, Cd BCF and Cd TF pointed out the existence of restrictive mechanisms on Cd translocation from lower plant parts to upper plant parts, mainly under highest $\mathrm{Cd}$ exposure}

Cadmium concentration increased in all plant tissues of $P$. maximum due to $\mathrm{Cd}$ exposure (Fig. 4). However, the $\mathrm{Cd}$ concentrations measured in the leaves (Figs. 4A-D) were similar to those observed in the stems (Fig. 4E) and much lower in relation to basal node and roots (Figs. 4F-G) of $P$. maximum exposed to the highest available $\mathrm{Cd}$ concentrations in the Oxisol. The leaves I, II, III and other leaves presented Cd concentrations lower than $30 \mathrm{mg} \mathrm{kg}^{-1}$ DW in both growth periods (Figs. 4A-D), whereas Cd concentrations in the basal node and roots of $P$. maximum exposed to the available $\mathrm{Cd}$ concentration of $10.91 \mathrm{mg} \mathrm{kg}^{-1}$ soil were higher than 100 and $70 \mathrm{mg} \mathrm{kg}^{-1} \mathrm{DW}$, respectively (Figs. 4F-G). There was effect of the growth period on $\mathrm{Cd}$ concentration only for the stems of $P$. maximum grown with the available $\mathrm{Cd}$ concentration of $10.91 \mathrm{mg}$ $\mathrm{kg}^{-1}$ soil, in which $\mathrm{Cd}$ concentration in the regrowth period was $86 \%$ higher compared to growth period (Fig. 4E).

In general, the highest $\mathrm{Cd} B C F$ s values were found in $P$. maximum exposed to the available $\mathrm{Cd}$ concentration of $2.86 \mathrm{mg} \mathrm{kg}^{-1}$ soil (Figs. 5A-E). The Cd BCFs values in the leaf II and stems of $P$. maximum exposed to the available $\mathrm{Cd}$ concentration of $2.86 \mathrm{mg} \mathrm{kg}^{-1}$ soil were higher in the regrowth compared to growth period (Figs. 5B and 5E). Cadmium BCFs remained higher than 1 when the grass was exposed to the available Cd concentrations of $2.86,5.93$ and $10.91 \mathrm{mg} \mathrm{kg}^{-1}$ soil, regardless of the aerial plant tissue. Similarly to which was observed for $\mathrm{Cd} \mathrm{BCFs,} \mathrm{the} \mathrm{highest} \mathrm{Cd}$ TF was found in $P$. maximum exposed to the available $\mathrm{Cd}$ concentration of $2.86 \mathrm{mg} \mathrm{kg}^{-1}$ soil, and Cd TF also remained $>1$ when this grass was exposed to the available Cd concentrations of 2.86, 5.93 and $10.91 \mathrm{mg} \mathrm{kg}^{-1}$ soil in the Oxisol (Fig. 5F).

\section{Both Cd exposure and growth period affected nutrients concentration and NUE of P. maximum, but the decreased NUEs Cd-induced did not negatively correlate with Cd BCF}

In general, the concentrations of $\mathrm{P}, \mathrm{K}, \mathrm{S}$ and $\mathrm{Cu}$ increased, and the concentrations of $\mathrm{B}$ and $\mathrm{Mn}$ tended to decrease in the plant tissues of $P$. maximum exposed to the highest available $\mathrm{Cd}$ concentrations (Table 2). Phosphorus, $\mathrm{Mg}, \mathrm{S}$ and Cu presented higher concentrations in the tissues collected at the end of the growth compared to regrowth period, whereas $\mathrm{K}, \mathrm{Fe}$ and $\mathrm{Mn}$ were found in higher concentrations in the regrowth period. $\mathrm{P}, \mathrm{K}, \mathrm{Ca}, \mathrm{Mg}$ and $\mathrm{S}$ tended to follow an increasing gradient of concentration in the sequence: roots < 
basal node $<$ stems < leaves. On the other hand, the higher concentrations of $B, C u$ and Fe were found in the roots, and $\mathrm{Mn}$ and $\mathrm{Zn}$ in the basal node of $P$. maximum. 
Table 2

Effect of the available $\mathrm{Cd}$ concentrations, growth periods, plant tissues and their interactions on macronutrients and micronutrients concentrations of Panicum maximum established in non-polluted and Cd-polluted Oxisol

\begin{tabular}{|c|c|c|c|c|c|c|c|c|c|c|}
\hline \multirow{2}{*}{$\begin{array}{l}\text { Available Cd } \\
\text { concentrations } \\
\text { (mg } \mathrm{kg}^{-1} \text { soil) }\end{array}$} & $\mathbf{P}$ & $\mathrm{K}$ & $\mathrm{Ca}$ & $\mathrm{Mg}$ & $s$ & B & $\mathrm{Cu}$ & $\mathrm{Fe}$ & Mn & $\mathrm{Zn}$ \\
\hline & \multicolumn{5}{|c|}{$\mathrm{g} \mathrm{kg}^{-1} \mathrm{DW}$} & \multicolumn{5}{|c|}{$\mathrm{mg} \mathrm{kg}^{-1} \mathrm{DW}$} \\
\hline 0.04 (control) & $\begin{array}{l}0.58 \\
c\end{array}$ & $\begin{array}{l}15.88 \\
b\end{array}$ & $\begin{array}{l}3.64 \\
a b\end{array}$ & $\begin{array}{l}2.11 \\
a b\end{array}$ & $\begin{array}{l}0.85 \\
b\end{array}$ & $\begin{array}{l}10.06 \\
a\end{array}$ & $\begin{array}{l}3.16 \\
c\end{array}$ & 70.2 & $\begin{array}{l}612.5 \\
\mathrm{a}\end{array}$ & 32.5 \\
\hline 2.86 & $\begin{array}{l}0.64 \\
b\end{array}$ & $\begin{array}{l}16.64 \\
b\end{array}$ & $\begin{array}{l}3.91 \\
a\end{array}$ & $\begin{array}{l}2.38 \\
a\end{array}$ & $\begin{array}{l}0.93 \\
b\end{array}$ & $\begin{array}{l}10.06 \\
a\end{array}$ & $\begin{array}{l}3.92 \\
b\end{array}$ & 239.0 & $\begin{array}{l}442.3 \\
\mathrm{~b}\end{array}$ & 36.4 \\
\hline 5.93 & $\begin{array}{l}0.72 \\
a\end{array}$ & $\begin{array}{l}17.25 \\
b\end{array}$ & $\begin{array}{l}3.70 \\
a b\end{array}$ & $\begin{array}{l}2.37 \\
a\end{array}$ & $\begin{array}{l}0.97 \\
b\end{array}$ & $\begin{array}{l}8.22 \\
b\end{array}$ & $\begin{array}{l}4.07 \\
b\end{array}$ & 75.2 & $\begin{array}{l}541.9 \\
a b\end{array}$ & 30.7 \\
\hline 10.91 & $\begin{array}{l}0.71 \\
a\end{array}$ & $\begin{array}{l}23.20 \\
\mathrm{a}\end{array}$ & $\begin{array}{l}3.35 \\
b\end{array}$ & $\begin{array}{l}1.88 \\
b\end{array}$ & $\begin{array}{l}1.71 \\
a\end{array}$ & $\begin{array}{l}8.58 \\
a b\end{array}$ & $\begin{array}{l}5.94 \\
a\end{array}$ & 69.4 & $\begin{array}{l}474.6 \\
b\end{array}$ & 36.0 \\
\hline \multicolumn{11}{|l|}{$\begin{array}{l}\text { Growth } \\
\text { periods }\end{array}$} \\
\hline First (growth) & $\begin{array}{l}0.71 \\
a\end{array}$ & $\begin{array}{l}16.68 \\
b\end{array}$ & 3.64 & $\begin{array}{l}2.41 \\
a\end{array}$ & $\begin{array}{l}1.53 \\
a\end{array}$ & 9.60 & $\begin{array}{l}5.06 \\
a\end{array}$ & $54.0 \mathrm{~b}$ & $\begin{array}{l}296.5 \\
b\end{array}$ & 30.8 \\
\hline $\begin{array}{l}\text { Second } \\
\text { (regrowth) }\end{array}$ & $\begin{array}{l}0.62 \\
b\end{array}$ & $\begin{array}{l}19.80 \\
a\end{array}$ & 3.66 & $\begin{array}{l}1.96 \\
b\end{array}$ & $\begin{array}{l}0.69 \\
\mathrm{~b}\end{array}$ & 8.87 & $\begin{array}{l}3.49 \\
b\end{array}$ & $\begin{array}{l}173.0 \\
\mathrm{a}\end{array}$ & $\begin{array}{l}739.2 \\
a\end{array}$ & 36.8 \\
\hline \multicolumn{11}{|l|}{ Plant tissues } \\
\hline Leaf I & $\begin{array}{l}0.71 \\
a b\end{array}$ & $\begin{array}{l}18.86 \\
a b\end{array}$ & $\begin{array}{l}3.31 \\
c\end{array}$ & $\begin{array}{l}1.71 \\
d\end{array}$ & $\begin{array}{l}1.10 \\
a b\end{array}$ & $\begin{array}{l}10.04 \\
b c\end{array}$ & $\begin{array}{l}4.78 \\
\mathrm{~cd}\end{array}$ & $\begin{array}{l}173.6 \\
b c\end{array}$ & $\begin{array}{l}433.0 \\
\text { bc }\end{array}$ & $\begin{array}{l}30.3 \\
b\end{array}$ \\
\hline Leaf II & $\begin{array}{l}0.67 \\
\mathrm{bc}\end{array}$ & $\begin{array}{l}18.19 \\
a b\end{array}$ & $\begin{array}{l}3.78 \\
\mathrm{bc}\end{array}$ & $\begin{array}{l}1.87 \\
\mathrm{~cd}\end{array}$ & $\begin{array}{l}1.15 \\
a\end{array}$ & $\begin{array}{l}9.95 \\
\mathrm{bc}\end{array}$ & $\begin{array}{l}4.28 \\
\mathrm{~cd}\end{array}$ & $73.0 \mathrm{c}$ & $\begin{array}{l}497.9 \\
a b\end{array}$ & $\begin{array}{l}27.9 \\
b\end{array}$ \\
\hline Leaf III & $\begin{array}{l}0.61 \\
\mathrm{~cd}\end{array}$ & $\begin{array}{l}16.24 \\
b\end{array}$ & $\begin{array}{l}4.38 \\
a\end{array}$ & $\begin{array}{l}2.19 \\
b c\end{array}$ & $\begin{array}{l}1.22 \\
a\end{array}$ & $\begin{array}{l}10.70 \\
b\end{array}$ & $\begin{array}{l}3.76 \\
\mathrm{~cd}\end{array}$ & $68.7 c$ & $\begin{array}{l}574.9 \\
a b\end{array}$ & $\begin{array}{l}26.0 \\
b\end{array}$ \\
\hline Other leaves & $\begin{array}{l}0.75 \\
a\end{array}$ & $\begin{array}{l}18.19 \\
a b\end{array}$ & $\begin{array}{l}4.21 \\
a b\end{array}$ & $\begin{array}{l}2.56 \\
a b\end{array}$ & $\begin{array}{l}1.23 \\
a\end{array}$ & $\begin{array}{l}10.26 \\
b\end{array}$ & $\begin{array}{l}4.88 \\
c\end{array}$ & $\begin{array}{l}105.4 \\
c\end{array}$ & $\begin{array}{l}557.6 \\
a b\end{array}$ & $\begin{array}{l}39.4 \\
b\end{array}$ \\
\hline Stems & $\begin{array}{l}0.56 \\
\mathrm{de}\end{array}$ & $\begin{array}{l}19.72 \\
a\end{array}$ & $\begin{array}{l}2.57 \\
d\end{array}$ & $\begin{array}{l}2.60 \\
a\end{array}$ & $\begin{array}{l}0.87 \\
c\end{array}$ & $\begin{array}{l}5.21 \\
d\end{array}$ & $\begin{array}{l}3.66 \\
d\end{array}$ & $\begin{array}{l}146.6 \\
c\end{array}$ & $\begin{array}{l}525.8 \\
a b\end{array}$ & $\begin{array}{l}45.4 \\
b\end{array}$ \\
\hline Basal node & $\begin{array}{l}0.50 \\
e^{-1}\end{array}$ & $\begin{array}{l}6.43 \\
c\end{array}$ & $e^{1.75}$ & $e^{1.18}$ & $\begin{array}{l}0.73 \\
\mathrm{c}\end{array}$ & $\begin{array}{l}7.15 \\
\text { cd }\end{array}$ & $\begin{array}{l}6.21 \\
b\end{array}$ & $\begin{array}{l}748.4 \\
\mathrm{~b}\end{array}$ & $\begin{array}{l}606.5 \\
\mathrm{a}\end{array}$ & $\begin{array}{l}284.9 \\
a\end{array}$ \\
\hline Roots & $\begin{array}{l}0.37 \\
f\end{array}$ & $\begin{array}{l}3.08 \\
d\end{array}$ & $\mathrm{e}^{1.32}$ & $\begin{array}{l}0.73 \\
f\end{array}$ & $\begin{array}{l}0.92 \\
b c\end{array}$ & $\begin{array}{l}18.16 \\
a\end{array}$ & $\begin{array}{l}9.08 \\
a\end{array}$ & $\begin{array}{l}3516.4 \\
a\end{array}$ & $\begin{array}{l}336.8 \\
c\end{array}$ & $\begin{array}{l}29.8 \\
b\end{array}$ \\
\hline
\end{tabular}

Different letters within the same column indicate differences between means (Tukey test, $p<0.05$ ); significant effects for the main factors and for interaction between them are indicated with asterisks at the level of ${ }^{*} p<0.05$, ** $p<0.01$ and ${ }^{* * *} p<0.001$; ns $=$ not significant 


\begin{tabular}{|c|c|c|c|c|c|c|c|c|c|c|}
\hline \multirow{2}{*}{$\begin{array}{l}\text { Available Cd } \\
\text { concentrations } \\
\text { (mg } \mathrm{kg}^{-1} \text { soil) }\end{array}$} & $P$ & $\mathrm{~K}$ & $\mathrm{Ca}$ & $\mathrm{Mg}$ & $\mathrm{S}$ & B & $\mathrm{Cu}$ & $\mathrm{Fe}$ & $M n$ & $\mathrm{Zn}$ \\
\hline & \multicolumn{5}{|c|}{$\mathrm{g} \mathrm{kg}^{-1} \mathrm{DW}$} & \multicolumn{5}{|c|}{$\mathrm{mg} \mathrm{kg}^{-1} \mathrm{DW}$} \\
\hline $\begin{array}{l}\text { Cd } \\
\text { concentration }\end{array}$ & $\star \star \star$ & $\star \star \star$ & $\star \star$ & $\star \star \star$ & $\star \star \star$ & $\star \star$ & $\star \star \star$ & ns & $\star \star \star$ & ns \\
\hline Growth period & $\star \star \star$ & $\star \star \star *$ & ns & $\star \star \star$ & $\star \star \star *$ & ns & $\star \star \star$ & $\star \star \star *$ & $\star \star \star$ & ns \\
\hline Plant tissue & 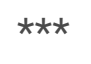 & $\star \star \star *$ & $\star \star \star \star$ & $\star \star \star$ & $\star \star \star *$ & $\star \star \star *$ & $\star \star \star *$ & $\star \star \star *$ & $\star * \star$ & $\star \star \star *$ \\
\hline $\begin{array}{l}\text { Cd } \\
\text { concentration } \\
\times \text { Growth } \\
\text { period }\end{array}$ & 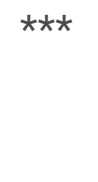 & $\star \star \star \star$ & ns & ns & $\star \star \star *$ & ns & $\star \star \star ~$ & ns & $\star \star \star$ & ns \\
\hline $\begin{array}{l}\text { Cd } \\
\text { concentration } \\
\times \text { Plant tissue }\end{array}$ & ns & $\star \star *$ & ns & * & ns & $\star \star \star$ & ** & $\star \star \star *$ & ns & $\star \star *$ \\
\hline $\begin{array}{l}\text { Growth period } \\
\times \text { Plant tissue }\end{array}$ & $\star \star \star$ & $\star \star *$ & ** & ** & $\star \star \star *$ & ns & * & ns & $\star \star \star$ & $\mathrm{ns}$ \\
\hline $\begin{array}{l}\text { Cd } \\
\text { concentration } \\
\times \text { Growth } \\
\text { period } \times \text { Plant } \\
\text { tissue }\end{array}$ & ns & ns & ns & ns & ns & ns & ns & ns & ns & ns \\
\hline $\begin{array}{l}\text { Different letters } \\
\text { significant effec } \\
\text { the level of } * p<\end{array}$ & for & sar & lum & $\begin{array}{l}\text { dica } \\
\text { Ifor }\end{array}$ & iffe & etw & $\begin{array}{l}\text { near } \\
n \text { ar }\end{array}$ & $\begin{array}{l}\text { uke } \\
\text { lica }\end{array}$ & $\begin{array}{l}0<0 \\
\text { ר ast }\end{array}$ & i \\
\hline
\end{tabular}

Cadmium exposure reduced the NUE of P, K, Ca, S, Cu, Fe, Mn and Zn compared to control (Table 3). However, there was no significant correlation between the NUE of each nutrient and the Cd BCFs (Supplementary Fig. 1). Only Mn-NUE was higher in the growth compared to regrowth period of $P$. maximum, whereas $\mathrm{P}, \mathrm{Ca}, \mathrm{Mg}, \mathrm{S}, \mathrm{B}, \mathrm{Cu}$ and $\mathrm{Zn}$ presented higher NUEs at the end of the regrowth period. The highest NUEs for P, K, Ca, Mg, S, B, Cu, Mn and Zn were found in the roots (Table 3), followed by the lower plant parts and then the upper plant parts, differently from that observed for nutrients concentration (Table 2), when we analyzed the NUE of each nutrient within the plant tissues of $P$. maximum. 
Table 3

Effect of the available $\mathrm{Cd}$ concentrations, growth periods, plant tissues and their interactions on macronutrients and micronutrients use efficiency (NUE) by Panicum maximum established in non-polluted and Cd-polluted Oxisol

\begin{tabular}{|c|c|c|c|c|c|c|c|c|c|c|}
\hline \multirow{2}{*}{$\begin{array}{l}\text { Available Cd } \\
\text { concentrations ( } \mathrm{mg} \mathrm{kg}^{-1} \\
\text { soil) }\end{array}$} & $\mathbf{P}$ & K & $\mathrm{Ca}$ & $\mathrm{Mg}$ & $S$ & B & $\mathrm{Cu}$ & $\mathrm{Fe}$ & Mn & Zn \\
\hline & \multicolumn{5}{|c|}{$\mathrm{g}^{2} \mathrm{mg}^{-1}$} & \multicolumn{5}{|c|}{$\mathrm{g}^{2} \mathrm{ng}^{-1}$} \\
\hline 0.04 (control) & $\begin{array}{l}1.13 \\
a\end{array}$ & $\begin{array}{l}0.04 \\
a b\end{array}$ & $\begin{array}{l}0.17 \\
a b\end{array}$ & 0.28 & $\begin{array}{l}0.85 \\
a\end{array}$ & 80 & $\begin{array}{l}200 \\
a\end{array}$ & $\begin{array}{l}11 \\
a\end{array}$ & $\begin{array}{l}15 \\
a b\end{array}$ & $\begin{array}{l}19 \\
a b\end{array}$ \\
\hline 2.86 & $\begin{array}{l}1.00 \\
a\end{array}$ & $\begin{array}{l}0.06 \\
a\end{array}$ & $\begin{array}{l}0.19 \\
a\end{array}$ & 0.34 & $\begin{array}{l}0.81 \\
a\end{array}$ & 80 & $\begin{array}{l}170 \\
a b\end{array}$ & $\begin{array}{l}11 \\
a\end{array}$ & $\begin{array}{l}19 \\
a\end{array}$ & $\begin{array}{l}20 \\
\mathrm{a}\end{array}$ \\
\hline 5.93 & $\begin{array}{l}0.82 \\
b\end{array}$ & $\begin{array}{l}0.03 \\
a b\end{array}$ & $\begin{array}{l}0.16 \\
a b\end{array}$ & 0.22 & $\begin{array}{l}0.73 \\
a\end{array}$ & 80 & $\begin{array}{l}140 \\
b\end{array}$ & $\begin{array}{l}8 \\
a b\end{array}$ & $\begin{array}{l}13 \\
b\end{array}$ & $\begin{array}{l}16 \\
b\end{array}$ \\
\hline 10.91 & $\begin{array}{l}0.71 \\
b\end{array}$ & $\begin{array}{l}0.01 \\
b\end{array}$ & $\begin{array}{l}0.14 \\
b\end{array}$ & 0.22 & $\begin{array}{l}0.46 \\
b\end{array}$ & 70 & $\begin{array}{l}90 \\
\mathrm{c}\end{array}$ & $\begin{array}{l}6 \\
b\end{array}$ & $7 c$ & $\begin{array}{l}11 \\
\mathrm{c}\end{array}$ \\
\hline
\end{tabular}

Growth periods

$\begin{array}{llllllllllll}\text { First (growth) } & \begin{array}{llllll}0.62 \\ \mathrm{~b}\end{array} & 0.02 & \begin{array}{l}0.12 \\ \mathrm{~b}\end{array} & \begin{array}{l}0.18 \\ \mathrm{~b}\end{array} & \begin{array}{l}0.34 \\ \mathrm{~b}\end{array} & \begin{array}{l}50 \\ \mathrm{~b}\end{array} & \begin{array}{l}100 \\ \mathrm{~b}\end{array} & 9 & \begin{array}{l}17 \\ \mathrm{a}\end{array} & \begin{array}{l}15 \\ \mathrm{~b}\end{array} \\ \text { Second (regrowth) } & 1.21 & 0.04 & \begin{array}{l}0.21 \\ \mathrm{a}\end{array} & \begin{array}{lllll}0.35 \\ \mathrm{a}\end{array} & & \begin{array}{l}1.08 \\ \mathrm{a}\end{array} & \begin{array}{l}110 \\ \mathrm{a}\end{array} & \begin{array}{l}200 \\ \mathrm{a}\end{array} & \begin{array}{l}\mathrm{a} \\ \mathrm{a}\end{array} & \begin{array}{l}10 \\ \mathrm{~b}\end{array} & \begin{array}{l}18 \\ \mathrm{a}\end{array}\end{array}$

Plant tissues

$\begin{array}{lllllllllll}\text { Leaf I } & 0.74 & 0.03 & 0.16 & 0.33 & 0.60 & 50 & 120 & 8 & 1 \mathrm{~b} & 18 \\ & \mathrm{bc} & \mathrm{b} & \mathrm{b} & \mathrm{b} & \mathrm{cd} & \mathrm{bc} & \mathrm{bc} & \mathrm{b} & & \mathrm{bcd} \\ \text { Leaf II } & 0.60 & 0.02 & 0.10 & 0.21 & 0.46 & 40 & 100 & 6 & 1 \mathrm{~b} & 14 \\ & \mathrm{c} & \mathrm{b} & \mathrm{b} & \mathrm{b} & \mathrm{cd} & \mathrm{bc} & \mathrm{bc} & \mathrm{bc} & & \mathrm{bcd} \\ \text { Leaf III } & 0.31 & 0.01 & 0.04 & 0.09 & 0.20 & 10 & 50 & 2 \mathrm{c} & 1 \mathrm{~b} & 7 \\ & \mathrm{c} & \mathrm{b} & \mathrm{b} & \mathrm{b} & \mathrm{d} & \mathrm{c} & \mathrm{c} & & & \mathrm{cd}\end{array}$

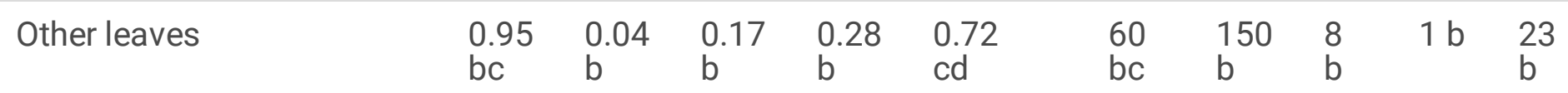

Stems

$\begin{array}{llllllllll}1.98 & 0.07 & 0.35 & 0.41 & 1.58 & 220 & 320 & 22 & 1 \mathrm{~b} & 20 \\ \mathrm{~b} & \mathrm{~b} & \mathrm{~b} & \mathrm{~b} & \mathrm{~b} & \mathrm{a} & \mathrm{a} & \mathrm{a} & & \mathrm{bc}\end{array}$

Basal node

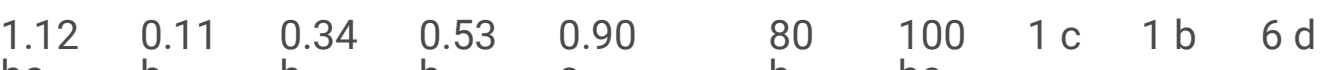

$\begin{array}{lllllll}\text { bc } & \text { b } & \text { b } & \text { b } & c & \text { b } & \text { bc }\end{array}$

Roots

$\begin{array}{llll}7.18 & 1.19 & 2.16 & 5.37 \\ \mathrm{a} & \mathrm{a} & \mathrm{a} & \mathrm{a}\end{array}$

3.14

180340

$\begin{array}{lll}3 & 12 & 89\end{array}$

a $\quad$ a $\quad$ bc $\quad$ a $\quad$ a

Statistical significance

Cd concentration

Different letters within the same column indicate differences between means (Tukey test, $p<0.05$ ); significant effects for the main factors and for interaction between them are indicated with asterisks at the level of ${ }^{*} p<0.05,{ }^{* *} p<0.01$ and ${ }^{* * *} p<0.001 ; n s=$ not significant 


\begin{tabular}{|c|c|c|c|c|c|c|c|c|c|c|}
\hline \multirow{2}{*}{$\begin{array}{l}\text { Available Cd } \\
\text { concentrations ( } \mathrm{mg} \mathrm{kg}^{-1} \\
\text { soil) }\end{array}$} & $P$ & $\mathrm{~K}$ & $\mathrm{Ca}$ & $\mathrm{Mg}$ & $\mathrm{S}$ & B & $\mathrm{Cu}$ & $\mathrm{Fe}$ & Mn & $\mathrm{Zn}$ \\
\hline & \multicolumn{5}{|c|}{$\mathrm{g}^{2} \mathrm{mg}^{-1}$} & \multicolumn{5}{|c|}{$\mathrm{g}^{2} \mathrm{ng}^{-1}$} \\
\hline Growth period & $\star \star \star$ & ns & $\star \star \star$ & $\star \star \star$ & $\star \star \star$ & $\star \star \star$ & $\star \star \star$ & $\star \star \star$ & $\star \star \star$ & $\star \star$ \\
\hline Plant tissue & 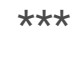 & $\star \star \star$ & $\star \star \star *$ & $\star \star \star ~$ & 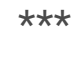 & $\star \star \star *$ & $\star \star \star$ & ns & $\star \star \star$ & $\star \star \star$ \\
\hline $\begin{array}{l}\text { Cd concentration } \times \text { Growth } \\
\text { period }\end{array}$ & $\star \star \star$ & ns & $\star \star \star ~$ & * & ns & * & ns & $\star \star \star$ & $\star \star \star$ & $\star \star \star$ \\
\hline $\begin{array}{l}\text { Cd concentration } \times \text { Plant } \\
\text { tissue }\end{array}$ & $\mathrm{ns}$ & $\mathrm{ns}$ & $\mathrm{ns}$ & ns & $\mathrm{ns}$ & $\mathrm{ns}$ & * & $\mathrm{ns}$ & $\mathrm{ns}$ & ns \\
\hline $\begin{array}{l}\text { Growth period } \times \text { Plant } \\
\text { tissue }\end{array}$ & $\star \star \star$ & $\star \star \star$ & 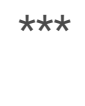 & $\star \star \star$ & 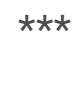 & $\star \star \star$ & $\star \star \star *$ & $\star \star \star$ & $\star \star \star$ & * \\
\hline $\begin{array}{l}\text { Cd concentration } \times \text { Growth } \\
\text { period } \times \text { Plant tissue }\end{array}$ & ns & ns & ns & ns & ns & ns & ns & ns & ns & ns \\
\hline \multicolumn{11}{|c|}{$\begin{array}{l}\text { Different letters within the same column indicate differences between means (Tukey test, } p<0.05 \text { ); } \\
\text { significant effects for the main factors and for interaction between them are indicated with asterisks at } \\
\text { the level of }{ }^{*} p<0.05, * \star p<0.01 \text { and } * \star \star p<0.001 \text {; } n=\text { not significant }\end{array}$} \\
\hline
\end{tabular}

\section{Discussion}

Cadmium effect on biomass yield and tillering of P. maximum used for phytoextraction in mildly polluted Oxisol along of two successive shoot growths

The exposure of $P$. maximum to the highest $\mathrm{Cd}$ concentrations decreased both leaves and stems biomass yield in the growth period (Figs. 2A-B), but there was no reduction on the number of tillers per plant in this growth period (Fig. 3A). Thereby, the reduction observed on shoot biomass yield can be attributed to a Cdinduced reduced number of leaves and shortening of stems and leaves (Supplementary Fig. 2). As gibberellins regulate the stem elongation rate in grasses (Zhang et al. 2016), Cd may have repressed gibberellins synthesis in P. maximum by affecting the KNOTTED1- like homeodomain (KNOX) proteins. KNOX proteins either activate or repress gibberellins synthesis genes, modifying levels of active gibberellins in the meristems and boundary regions of grasses (Pautler et al. 2013), which is the tillers initiation region (Chrysler 1906). Cadmium-induced changes on shoot meristematic region are also pointed out as a factor to reduce the number and length of leaves in plants of the family Poaceae. The decreased leaf length $\mathrm{Cd}$ induced in maize (Zea mays) grown in a mildly polluted soil (46.5 $\mathrm{mg} \mathrm{Cd} \mathrm{kg}^{-1}$ soil) was attributed to the lower number of meristematic cells, longer cell cycle duration and inhibition of cell elongation rate (Bertels et al. 2020).

During the regrowth, leaf and stem biomass yields of $P$. maximum exposed to the highest Cd concentrations did not differ from those plants of control treatment (Figs. 2A-B). These data indicate that $P$. maximum was able to cope with $\mathrm{Cd}$-induced stress under prolonged exposure by adapting its mechanisms of tolerance against Cd-induced stress (for a comprehensive review we suggest Rabêlo et al. 2021a). Such assumption is supported by the fact the number of tillers increased during plant regrowth compared to growth period 
(Fig. 3A), even the basal node (tiller initiation region) presenting high Cd concentrations (Fig. 4F). Moreover, there was not the trend of $\mathrm{Cd}$ accumulated in the basal node reduces the number of tillers during regrowth, differently from which was observed in the plant growth (Fig. 3B). It means that Cd probably is more harmful in the early stages of development of $P$. maximum grown in mildly polluted soils. Sunflower (Helianthus annuus) is also more susceptible to Cd-induced stress in the early stages of development because an uncontrolled Cd uptake that results in high Cd concentrations in its plant tissues (De Maria et al. 2013). However, as $\mathrm{Cd}$ concentrations in the leaf and stem tissues collected at the end of the growth and regrowth period were similar (Figs. 4A-E), other factors than Cd concentrations in the plant tissues limited the growth of $P$. maximum exposed to the highest $\mathrm{Cd}$ concentration in the first growth.

Interestingly, a decrease in $\mathrm{Mg}$ concentration in the leaves has been associated with plant protection against Cd-induced stress under prolonged exposure by improving the action of the antioxidant system (Chou et al. 2011; Hermans et al. 2011). Such mechanism possibly was employed by $P$. maximum, since lower $\mathrm{Mg}$ concentrations were observed in the leaves compared to stems, in the plants exposed to the highest $\mathrm{Cd}$ concentration compared to the other Cd concentrations, and in the regrowth compared to growth period (Table 2). This conferred higher tolerance to plants exposed to the highest $\mathrm{Cd}$ concentration in the regrowth period, but not in the growth period. Other nutritional adjustments occurred in $P$. maximum under $\mathrm{Cd}$ exposure (Table 2) and probably contributed for a higher Cd tolerance in the regrowth compared to growth period. Such nutritional adjustments are addressed in the next session.

As the number of tillers of $P$. maximum was higher in the regrowth compared to growth period (Fig. $3 \mathrm{~A})$, we can assume that plant density was higher in the regrowth period. Under such circumstance, plants show a clear increase in the stem fraction due to changes on carbohydrates allocation (Poorter et al. 2012). This explains why the stem biomass yield was higher and the leaf/stem ratio was lower in the regrowth than growth period (Figs. 2B and $3 \mathrm{C}$ ). Furthermore, there was no reduction on basal node biomass under $\mathrm{Cd}$ exposure (Fig. 2B), which may have contributed for the higher tillering in the regrowth period, since the tillers grow up from the axillary buds located at the basal node of the plant (Chrysler 1906). As observed for basal node biomass, the root biomass of $P$. maximum did not decrease due to Cd exposure (Fig. 2D), even this structure presenting high Cd concentrations (Fig. 4G). Maybe such result is associated to the fact that $P$. maximum preferentially accumulates $C d$ bound to cell wall in the root apoplast (Rabêlo et al. 2021b). In this case, the deleterious effects of $\mathrm{Cd}$ are more noticeable on root length and root surface than root weight (Rabêlo et al. 2020b), since the thickening of the roots due to lignification and suberization (Lux et al. 2011) can compensate the root weight.

\section{Distribution and accumulation of $\mathrm{Cd}$ within the plant tissues of P. maximum and its relationship with $\mathrm{Cd}$ phytoextraction efficiency and nutritional disorders}

Cadmium concentration increased in all plant tissues of $P$. maximum as a consequence of $\mathrm{Cd}$ exposure, but our results suggest the existence of restrictive mechanisms on $\mathrm{Cd}$ translocation from lower to upper plant parts because Cd concentration followed a decreasing gradient in the sequence: basal node > stems > leaves (Fig. 4). During Cd translocation in the xylem, $\mathrm{Cd}^{2+}$ ions interact with the cell walls of xylem vessels and are partly adsorb on them (Sterckeman and Thomine 2020). Furthermore, Cd accumulated in the leaves 
can be redistributed to other plant organs via phloem or even to roots where Cd could be excreted (Sterckeman and Thomine 2020). Thus, with exception of hyperaccumulators plants, a restriction on $\mathrm{Cd}$ translocation upwards is expected, mainly under higher $\mathrm{Cd}$ exposure. Cadmium translocation from roots to shoot was higher when $P$. maximum was grown in the Oxisol presenting the available $\mathrm{Cd}$ concentration of $2.86 \mathrm{mg} \mathrm{kg}^{-1}$ soil, but from this point there was a reduction on Cd TF (Fig. 5F). Our results are similar to those reported for lettuce (Lactuca sativa), spinach (Spinacia oleracea), cauliflower (Brassica oleracea) and oat (Avena sativa), in which Cd concentrations were higher in the shoots than roots when plants were grown on low Cd-polluted soil, but Cd concentrations in the roots became higher than of the shoots when these plants were grown on more polluted soil (John 1973). Similarly to Cd TF, the higher Cd BCFs were observed when $P$. maximum was exposed to the available $C d$ concentration of $2.86 \mathrm{mg} \mathrm{kg}^{-1}$ soil, and from this point the $\mathrm{Cd}$ BCFs decreased (Figs. 5A-E). Although the two factors remained higher than 1 under $\mathrm{Cd}$ exposure, $\mathrm{Cd}$ $\mathrm{TF}$ and $\mathrm{Cd} \mathrm{BCFs}$ decreased under the highest $\mathrm{Cd}$ concentrations in the Oxisol, indicating lowered phytoextraction efficiency in such conditions. The potential of phytoextraction tends to decrease when grass species are faced to more high $\mathrm{Cd}$ concentrations due to $\mathrm{Cd}$-induced toxicity, such as nutritional disorders (Rabêlo et al., 2021a).

Nutritional disorders are common in grasses exposed to Cd (Rabêlo and Borgo 2016), which can decrease Cd phytoextraction efficiency (Rabêlo et al. 2020a), even the lower NUE of P, K, Ca, S, Cu, Fe, Mn and Zn observed in P. maximum under $\mathrm{Cd}$ exposure (Table 3 ) did not negatively correlating with Cd BCF (Supplementary Fig. 1). Changes on nutrients' concentration and use are coupled to negative outcomes on the development of plants under $\mathrm{Cd}$ exposure, but there is evidence that nutritional adjustments are necessary for plants cope with Cd stress (for a review, see Carvalho et al. 2020). For instance, a reduction in leaves $\mathrm{Mg}$ concentration of plants exposed to $\mathrm{Cd}$ can improve the action of the antioxidant system (Chou et al. 2011; Hermans et al. 2011), making possible an increase on biomass yield (Carvalho et al. 2020), as observed in our study (Figs. 2A-B; Table 2). Another example is $\mathrm{K}$, which is involved on biomass allocation due its role on carbohydrates loading into the phloem for long-distance transport. Under lower $\mathrm{K}$ concentration sucrose export into the phloem is reduced (Cakmak et al. 1994), but increased $\mathrm{K}^{+}$may promote sugar unloading in sink tissues and speed the conversion of sucrose to synthetic metabolites (Conti and Geiger 1982), which allow biomass yield. Thus, the increase in the stem biomass yield induced by the higher plant density in the regrown P. maximum (Figs. 2B and $3 \mathrm{~A}$ ), especially in those plants exposed to the highest $\mathrm{Cd}$ concentrations, possibly is associated with the increased $\mathrm{K}$ concentrations verified in the stems, in the regrown plants and in the plants exposed to the highest Cd concentration (Table 2).

The concentrations of $\mathrm{P}, \mathrm{S}$ and $\mathrm{Cu}$ of $P$. maximum increased after $\mathrm{Cd}$ exposure (Table 2). Under high $\mathrm{P}$ concentrations, more $\mathrm{P}$ is accumulated in the root and may form insoluble phosphate precipitates with $\mathrm{Cd}$ in the cell wall and vacuoles, which prevents the transport of $\mathrm{Cd}$ to the protoplasm and xylem and inhibits the transport of Cd to the shoot (Guo et al., 2018). Indeed, Cd TF was reduced in P. maximum exposed to the available Cd concentrations of 5.93 and $10.91 \mathrm{mg} \mathrm{kg}^{-1}$ soil (Fig. 5F), where higher shoot $\mathrm{P}$ concentrations were found (Table 2). Plants under $\mathrm{Cd}$ exposure tends to uptake more $\mathrm{S}$ due its role on glutathione (GSH, $\mathrm{Y}^{-}$ Glu-Cys-Gly) and phytochelatins [PCs, (y-Glu-Cys) ${ }_{n}-$ Gly, with $\left.n=2-11\right]$ synthesis, which are peptides involved in plant tolerance against to $\mathrm{Cd}$-induced stress (for a comprehensive review we suggest Gill and Tuteja 
2011). Sulfur concentration was higher in the growth than regrowth period (Table 2), which makes sense, since $\mathrm{Cd}$ is more stored as chelates (e.g., PC-Cd) in the vacuoles of plants in the early stages of development, whereas other detoxification mechanisms (e.g., Cd bound to cell walls) are more employed under prolonged Cd exposure (Rabêlo et al. 2018, 2021a; Sterckeman and Thomine 2020). As an increase on antioxidant activity of $P$. maximum was speculated due to decreased $\mathrm{Mg}$ and increased $\mathrm{S}$ concentrations, an increase on Cu concentration due to Cd exposure, especially in the growth period (Table 2), makes sense since $\mathrm{Cu}$ is a cofactor of the enzyme superoxide dismutase (SOD, EC 1.15.1.1) (Gratão et al. 2005). Superoxide dismutases, such as the isoenzyme Cu/Zn-SOD, act as the first line of defense against reactive oxygen species by dismutating superoxide $\left(\mathrm{O}_{2}{ }^{-}\right)$in $\mathrm{H}_{2} \mathrm{O}_{2}$ (Gratão et al. 2005). In addition, the presence of $\mathrm{Cu} / \mathrm{Zn}-\mathrm{SOD}$ in the apoplast of spinach was positively correlated to sites of lignification (Ogawa et al. 1996). In this sense, is plausible to assume that the higher $\mathrm{Cu}$ concentration observed in the roots of $P$. maximum (Table 2) favored root lignification through the action of $\mathrm{Cu} / \mathrm{Zn}-\mathrm{SOD}$ in the root apoplast (main local of $\mathrm{Cd}$ storage in this species; Rabêlo et al. 2021b), which avoid a strong reduction on the root weight of plants exposed to the highest Cd concentrations in the Oxisol due to a root thickening (Fig. 2D).

The concentrations of $\mathrm{B}$ and $\mathrm{Mn}$ tended to decrease in the plant tissues of $P$. maximum exposed to the highest $\mathrm{Cd}$ concentrations, differently from which was observed for $\mathrm{P}, \mathrm{K}, \mathrm{S}$ and $\mathrm{Cu}$ (Table 2). In tomato (Solanum lycopersicum), Cd toxicity was related to B and $\mathrm{Mn}$ excess in leaves, in addition to the own $\mathrm{Cd}$ accumulation, since the symptoms of $\mathrm{Cd}$ toxicity in leaf tissues resembled those triggered by $\mathrm{B}$ and $\mathrm{Mn}$ excess (Carvalho et al. 2018). It is possible that $P$. maximum had decreased both $B$ and $\mathrm{Mn}$ concentrations in its tissues as a strategy of adaptation to Cd-induced stress, as no visual symptoms similar to those triggered by B and Mn excess were observed in our study (Supplementary Fig. 2).

The most part of nutrients' concentrations (Table 2), if not all, indicate that $P$. maximum cv. Massai poses strategies to cope with Cd-induced stress through nutritional adjustment (Carvalho et al., 2020). Even so, the NUE of $\mathrm{P}, \mathrm{K}, \mathrm{Ca}, \mathrm{S}, \mathrm{Cu}, \mathrm{Fe}, \mathrm{Mn}$ and $\mathrm{Zn}$ by P. maximum decreased under $\mathrm{Cd}$ exposure (Table 3 ). Lower NUEs in plants grown in polluted soils are expected due to phytotoxicity or internal adjustments which affect plant growth in such conditions (Baligar et al. 2001). Although there were no significant negative correlations between the NUEs and Cd BCFs (Supplementary Fig. 1), the data of NUE (Table 3) together with Cd TF and Cd BCFs (Fig. 5) support the statement of Rabêlo et al. (2021a), who described that the potential of phytoextraction of grass species faced to more high $\mathrm{Cd}$ concentrations tends to decrease due to $\mathrm{Cd}$-induced toxicity.

\section{Conclusions}

Cadmium toxicity was stronger in the early stages of development of $P$. maximum, not by reduce the tillering but to induce a stem shortening. Tillering was not compromised by the high $\mathrm{Cd}$ accumulation in the basal node of $P$. maximum, suggesting that the deleterious effects of $\mathrm{Cd}$ are more related with processes involved on stem elongation than tiller initiation from the axillary buds located in the basal node of $P$. maximum.

P. maximum presented a clear restriction on $\mathrm{Cd}$ transport upwards from basal node, which was the main local of $\mathrm{Cd}$ accumulation in this grass. Such restriction was more evident in the plants exposed to the 
highest Cd concentrations, and it was related to nutritional adjustments to cope with Cd-induced stress. Apparently, P, K, Mg, S and Cu are involved in processes that restrict Cd movement upwards and confer higher tolerance to $\mathrm{Cd}$ toxicity in $P$. maximum, but further studies are necessary to unravel the role of each one of these nutrients on $\mathrm{Cd}$ tolerance in this grass. The nutritional disorders $\mathrm{Cd}$-induced did not negatively correlate with Cd BCF, but the data of NUE, Cd TF and Cd BCF suggest that phytoextraction efficiency of $P$. maximum decreases from the available $C d$ concentration of $5.93 \mathrm{mg} \mathrm{kg}^{-1}$ soil.

\section{Declarations}

Author contributions FHSR performed the experiment and drafted the original manuscript. FHS helped to collect the experiment, process the plant material and analyze the data. JL helped in the data analysis and manuscript editing. LRFA revised the manuscript and supervised the study. All authors contributed to the critical review of the manuscript and approved its final version.

Funding This study was supported by São Paulo Research Foundation - FAPESP (grants \#2017/11299-8 and \#2018/07190-3).

\section{Compliance with ethical standards}

Conflict of interest The authors have no conflicts of interest to declare that are relevant to the content of this article.

Availability of data and material Not applicable.

Code availability Not applicable.

Ethics approval Not applicable.

Consent to participate Not applicable.

Consent for publication Not applicable.

\section{References}

1. Abreu CA, Abreu MF, Andrade JC, van Raij B, Andrade JC, Cantarella H (2001) Determination of copper, iron, manganese, zinc, cadmium, chromium, nickel and lead in soils using the DTPA solution at pH 7.3.. In: In: Quaggio JA (ed) Chemical Analysis for Fertility Evaluation of Tropical Soils. Instituto Agronômico de Campinas, Campinas, pp 240-250. (In Portuguese)

2. Ali H, Khan E, Sajad MA (2013) Phytoremediation of heavy metals-concepts and applications. Chemosphere 91:869-881. https://doi.org/10.1016/j.chemosphere.2013.01.075

3. Baligar VC, Fageria NK, He ZL (2001) Nutrient use efficiency in plants. Commun Soil Sci Plant Anal 32:921-950. https://doi.org/10.1081/CSS-100104098

4. Bataglia OC, van Raij B (1990) Efficiency of extractors in the determination of boron in soils. Rev Bras Cienc Solo 14:25-31. (In Portuguese) 
5. Bertels J, Huybrechts M, Hendrix S, Bervoets L, Cuypers A, Beemster GTS (2020) Cadmium inhibits cell cycle progression and specifically accumulates in the maize leaf meristem. J Exp Bot 71:6418-6428. https://doi.org/10.1093/jxb/eraa385

6. Cakmak I, Hengeler C, Marschner H (1994) Changes in phloem export of sucrose in leaves in response to phosphorus, potassium and magnesium deficiency in bean plants. J Exp Bot 45:1251-1257. https://doi.org/10.1093/jxb/45.9.1251

7. Carvalho MEA, Piotto FA, Franco MR, Borges KLR, Gaziola SA, Castro PRC, Azevedo RA (2018) Cadmium toxicity degree on tomato development is associated with disbalances in $B$ and Mn status at early stages of plant exposure. Ecotoxicology 27:1293-1302. https://doi.org/10.1007/s10646-018-1983-8

8. Carvalho MEA, Castro PRC, Kozak M, Azevedo RA (2020) The sweet side of misbalanced nutrients in cadmium stressed plants. Ann Appl Biol 176:275-284. https://doi.org/10.1111/aab.12569

9. Chou T-S, Chao Y-Y, Huang W-D, Hong C-Y, Kao C-H (2011) Effect of magnesium deficiency on antioxidant status and cadmium toxicity in rice seedlings. J Plant Physiol 168:1021-1030. https://doi.org/10.1016/j.jplph.2010.12.004

10. Chrysler MA (1906) The nodes of grasses. Bot Gaz 41:1-16. https://www.jstor.org/stable/2465514

11. Conti TR, Geiger DR (1982) Potassium nutrition and translocation in sugar beet. Plant Physiol 70:168172. https://doi.org/10.1104/pp.70.1.168

12. de Anicésio ÉCA, Monteiro FA (2019) Potassium affects the phytoextraction potential of Tanzania guinea grass under cadmium stress. Environ Sci Pollut Res 26:30472-30484. https://doi.org/10.1007/s11356-019-06191-x

13. de Anicésio ÉCA, Monteiro FA (2021) Potassium reduces oxidative stress in tanzania guinea grass under cadmium toxicity. Environ Sci Pollut Res. 10.1007/s11356-021-15620-9.. https://doi.org/10.1007/s11356-021-15620-9

14. De Maria S, Puschenreiter M, Rivelli AR (2013) Cadmium accumulation and physiological response of sunflower plants to Cd during the vegetative growing cycle. Plant Soil Environ 59:254-261. https://doi.org/10.17221/788/2012-PSE

15. EMBRAPA - Brazilian Agricultural Research Corporation (1997) Manual of Chemical Analysis of Soils, Plants and Fertilizers. Embrapa Comunicação para Transferência de Tecnologia, Brasília. (In Portuguese)

16. Farnezi MMM, Silva EB, Santos LL, Silva AC, Grazziotti PH, Prochnow JT (2020) Potential of grasses in phytolith production in soils contaminated with cadmium. Plants 9:109. https://doi.org/10.3390/plants9010109

17. Fujimaki S, Suzui N, Ishioka NS, Kawachi N, Ito S, Chino M, Nakamura S (2010) Tracing cadmium from culture to spikelet: noninvasive imaging and quantitative characterization of absorption, transport, and accumulation of cadmium in an intact rice plant. Plant Physiol 152:1796-1806. https://doi.org/10.1104/pp.109.151035

18. Garcez Neto AF, Gobbi KF, Silva J, Santos TM (2012) Tillering and biomass partitioning of Mombasa grass under nitrogen fertilization during regrowth. Rev Bras Zootecn 41:1824-1831. https://doi.org/10.1590/S1516-35982012000800004 
19. Gee GW, Bauder J (2002) Particle-size analysis.. In: In: Dane JH, Toop GC (eds) Methods of Soils Analysis. Part 4: Physical Methods. Soil Science Society of America, Madison, pp 255-293

20. Gill SS, Tuteja N (2011) Cadmium stress tolerance in crop plants - Probing the role of sulfur. Plant Signal Behav 6:215-222. https://doi.org/10.4161/psb.6.2.14880

21. Gratão PL, Polle A, Lea PJ, Azevedo RA (2005) Making the life of heavy metal-stressed plants a little easier. Funct Plant Biol 32:481-494. https://doi.org/10.1071/FP05016

22. Guo F, Ding C, Zhou Z, Huang G, Wang X (2018) Effects of combined amendments on crop yield and cadmium uptake in two cadmium contaminated soils under rice-wheat rotation. Ecotoxicol Environ Saf 148:303-310. https://doi.org/10.1016/j.ecoenv.2017.10.043

23. Hermans C, Chen J, Coppens F, Inzé D, Verbruggen N (2011) Low magnesium status in plants enhances tolerance to cadmium exposure. New Phytol 192:428-436. https://doi.org/10.1111/j.14698137.2011.03814.x

24. Houba VJG, Temminghoff EJM, Gaikhorst GA, Van Vark W (2000) Soil analysis procedures using 0.01 $\mathrm{M}$ calcium chloride as extraction reagent. Commun Soil Sci Plant Anal 31:1299-1396. https://doi.org/10.1080/00103620009370514

25. John MK (1973) Cadmium uptake by eight food crops as influenced by various soil levels of cadmium. Environ Pollut 4:7-15. https://doi.org/10.1016/0013-9327(73)90026-8

26. Lux A, Martinka M, Vaculík M, White PJ (2011) Root responses to cadmium in the rhizosphere: a review. J Exp Bot 62:21-37. https://doi.org/10.1093/jxb/erq281

27. Ogawa K, Kanematsu S, Asada K (1996) Intra- and extra-cellular localization of "cytosolic" CuZnsuperoxide dismutase in spinach leaf and hypocotyl. Plant Cell Physiol 37:790-799. https://doi.org/10.1093/oxfordjournals.pcp.a029014

28. Pautler M, Tanaka W, Hirano H-Y, Jackson D (2013) Grass Meristems I: Shoot apical meristem maintenance, axillary meristem determinacy and the floral transition. Plant Cell Physiol 54:302-312. https://doi.org/10.1093/pcp/pct025

29. Poorter H, Niklas KJ, Reich PB, Oleksyn J, Poot P, Mommer L (2012) Biomass allocation to leaves, stems and roots: meta-analyses of interspecific variation and environmental control. New Phytol 193:30-50. https://doi.org/10.1111/j.1469-8137.2011.03952.x

30. Rabêlo FHS, Borgo L (2016) Changes caused by heavy metals in micronutrient content and antioxidant system of forage grasses used for phytoremediation: an overview. Cienc Rural 46:1368-1375. https://doi.org/10.1590/0103-8478cr20151291

31. Rabêlo FHS, Azevedo RA, Monteiro FA (2017) Proper supply of S increases GSH synthesis in the establishment and reduces tiller mortality during the regrowth of Tanzania Guinea grass used for Cd phytoextraction. J Soils Sediments 17:1427-1436. https://doi.org/10.1007/s11368-016-1429-y

32. Rabêlo FHS, Fernie AR, Navazas A, Borgo L, Keunen E, Silva BKA, Cuypers A, Lavres J (2018) A glimpse into the effect of sulfur supply on metabolite profiling, glutathione and phytochelatins in Panicum maximum cv. Massai exposed to cadmium. Environ Exp Bot 151:76-88.

https://doi.org/10.1016/j.envexpbot.2018.04.003 
33. Rabêlo FHS, Lavres J, Ramires TG, Alleoni LRF (2020a) Diagnosis and recommendation integrated system and nutritional balance index reveal $\mathrm{Cd}$-induced nutritional disorders in Panicum maximum assayed for Cd phytoextraction. Bioremediat J 24:265-282.

https://doi.org/10.1080/10889868.2020.1841727

34. Rabêlo FHS, Borgo L, Merloti LF, Pylro VS, Navarrete AA, Mano RH, Thijs S, Vangronsveld J, Alleoni LRF (2020b) Effects of winter and summer conditions on Cd fractionation and bioavailability, bacterial communities and Cd phytoextraction potential of Brachiaria decumbens and Panicum maximum grown in a tropical soil. Sci Total Environ 728:138885. https://doi.org/10.1016/j.scitotenv.2020.138885

35. Rabêlo FHS, Vangronsveld J, Baker AJM, van der Ent A, Alleoni LRF (2021a) Are grasses really useful for the phytoremediation of potentially toxic trace elements? A Review. Front. Plant Sci 12:778275. https://doi.org/10.3389/fpls.2021.778275

36. Rabêlo FHS, Gaziola SA, Rossi ML, Silveira NM, Wójcik M, Bajguzf A, Piotrowska-Niczyporuk A, Lavres J, Linhares FS, Azevedo RA, Vangronsveld J, Alleoni LRF (2021b) Unraveling the mechanisms controlling $\mathrm{Cd}$ accumulation and $\mathrm{Cd}$ tolerance in Brachiaria decumbens and Panicum maximum under summer and winter weather conditions. Physiol Plant 173:20-44. https://doi.org/10.1111/ppl.13160

37. van Raij B, Andrade JC, Cantarella H, Quaggio JA (2001) Chemical Analysis for Fertility Evaluation of Tropical Soils. Instituto Agronômico de Campinas, Campinas. (In Portuguese)

38. Institute SAS (2008) SAS User's guide: Statistics. Version 9.2. Cary-United States of America. http://support.sas.com/software/92/

39. Siddiqi MY, Glass ADM (1981) Utilization index: A modified approach to the estimation and comparison of nutrient utilization efficiency in plants. J Plant Nutr 4:289-302.

https://doi.org/10.1080/01904168109362919

40. Sterckeman T, Thomine S (2020) Mechanisms of cadmium accumulation in plants. Crit Rev Plant Sci 39:322-359. https://doi.org/10.1080/07352689.2020.1792179

41. Tabatabai MA, Bremner JM (1970) An alkaline oxidation method for determination of total Sulphur in soils. Soil Sci Soc Am J 34:62-65. https://doi.org/10.2136/sssaj1970.03615995003400010019x

42. USDA - United States Department of Agriculture (1999) Soil Taxonomy: A Basic System of Soil Classification for Making and Interpreting Soil Surveys. U.S. Government Printing Office, Washington

43. USEPA - United States Environmental Protection Agency (1996) Method 3050B: Acid Digestion of Sediments, Sludges, and Soils, Revision 2. United States Environmental Protection Agency, Washington

44. USEPA - United States Environmental Protection Agency (2007) Method 3051A - Microwave Assisted Acid Digestion of Sediments, Sludges, Soils, and Oils. United States Environmental Protection Agency, Washington

45. Werner JC, Paulino VT, Cantarella H, Andrade NO, Quaggio JA (1997) Forages.. In: In: van Raij B, Cantarella H, Quaggio JA, Furlani AMC (eds) Fertilization and liming recommendations for the State of São Paulo. IAC, Campinas, pp 263-273. (In Portuguese)

46. Zhang N, Xie Y-D, Guo H-J, Zhao L-S, Xiong H-C, Gu J-Y, Li J-H, Kong F-Q, Sui L, Zhao Z-W, Zhao S-R, Liu $\mathrm{L}-\mathrm{X}$ (2016) Gibberellins regulate the stem elongation rate without affecting the mature plant height of a 
quick development mutant of winter wheat (Triticum aestivum L.). Plant Physiol Biochem 107:228-236. https://doi.org/10.1016/j.plaphy.2016.06.008

\section{Figures}

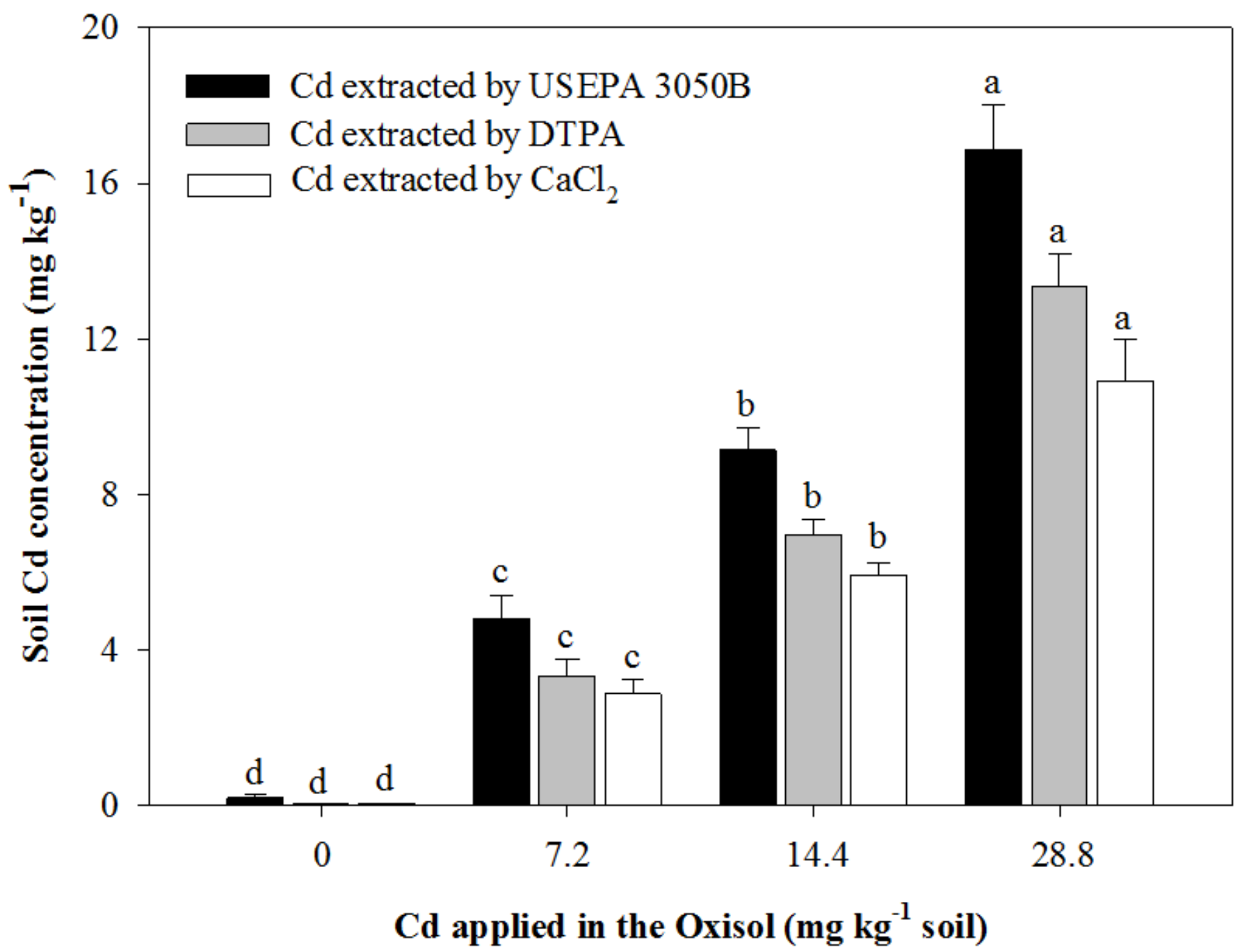

Figure 1

Cadmium concentration determined by USEPA 3050B, DTPA and $0.01 \mathrm{~mol} \mathrm{~L}^{-1} \mathrm{CaCl}_{2}$ methods in the Oxisol cultivated with Panicum maximum in function of the initial $\mathrm{Cd}$ doses applied. Distinct letters on the bars indicate difference between Cd concentrations for each Cd extractor $(n=4$, Tukey test, $\mathrm{p}<0.05)$. For the determination of Cd concentration extracted by DTPA $\left[0.005 \mathrm{~mol} \mathrm{~L}^{-1}\right.$ DTPA $+0.01 \mathrm{~mol} \mathrm{~L}^{-1} \mathrm{CaCl}_{2}+0.1 \mathrm{~mol} \mathrm{~L}^{-1}$ triethanolamine (TEA), at pH 7.3] (Abreu et al. 2001), $5 \mathrm{~g}$ of soil were dispersed in $20 \mathrm{~mL}$ of DTPA solution, shaken for $2 \mathrm{~h}$, and analyzed using an induced coupled plasma mass spectroscopy (ICP-MS, iCAP 7000 SERIES, Thermo Fisher Scientific, Waltham, USA). The concentrations of Cd extracted with $0.01 \mathrm{~mol} \mathrm{~L}^{-1}$ 
$\mathrm{CaCl}_{2}$ (Houba et al. 2000) were determined placing $4 \mathrm{~g}$ of soil in $50 \mathrm{~mL}$ centrifuge tubes and $40 \mathrm{~mL}$ of the extraction solution added. The samples were agitated for $2 \mathrm{~h}$ at $25^{\circ} \mathrm{C}$, centrifuged at $1800 \times \mathrm{g}$ for $10 \mathrm{~min}$, and filtered through filter paper $(0.45 \mu \mathrm{m})$. Then, the extracts were analyzed by ICP-MS
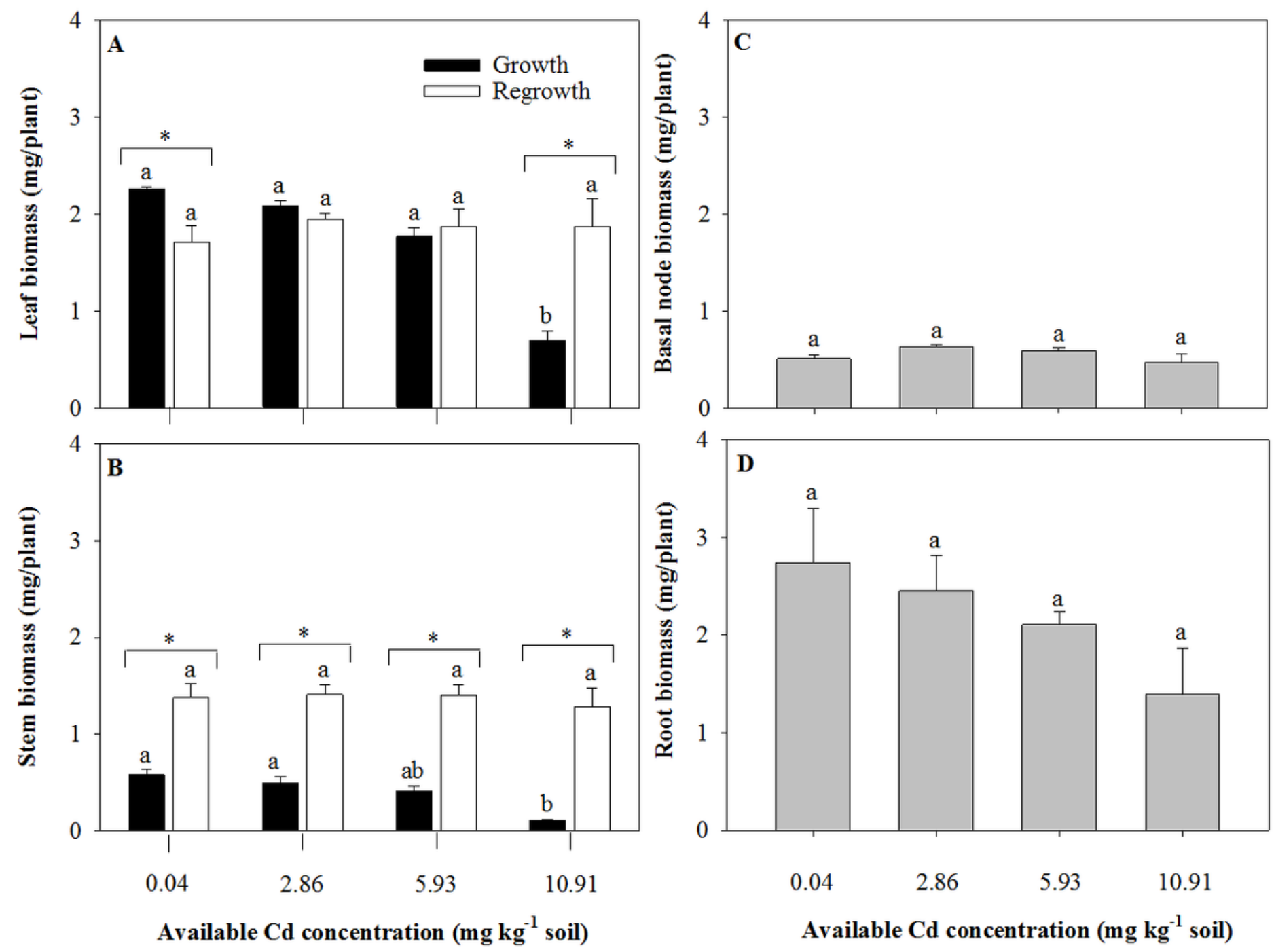

Figure 2

Biomass yield of leaves (A), stems (B), basal nodes (C) and roots (D) of Panicum maximum established in non-polluted and $\mathrm{Cd}$-polluted $\mathrm{Oxisol}$. Distinct letters on the bars indicate difference between $\mathrm{Cd}$ concentrations for each growth period of $P$. maximum $(n=4$, Tukey test, $\mathrm{p}<0.05)$. Asterisks represent differences at $p<0.05$ between growth periods within each Cd concentration (ANOVA, t-test) 


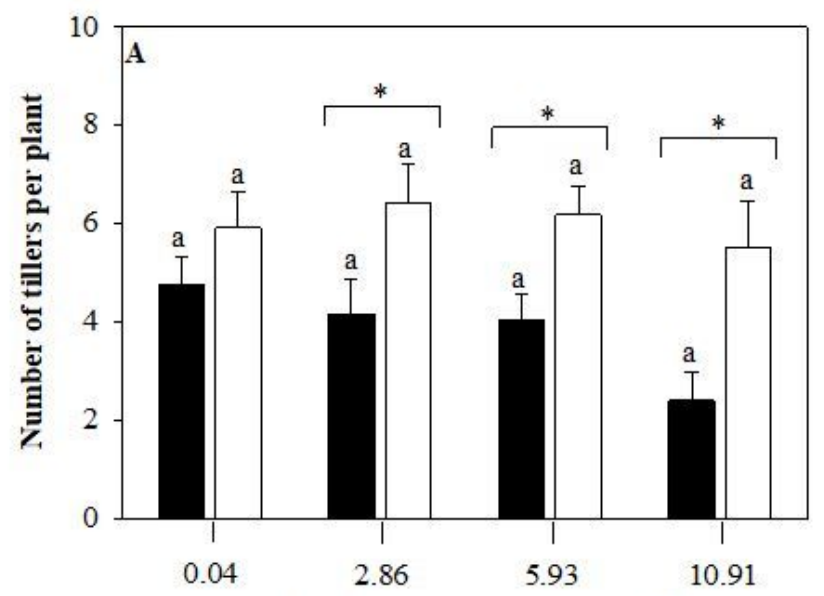

Available Cd concentration ( $\mathrm{mg} \mathrm{kg}^{-1}$ soil)
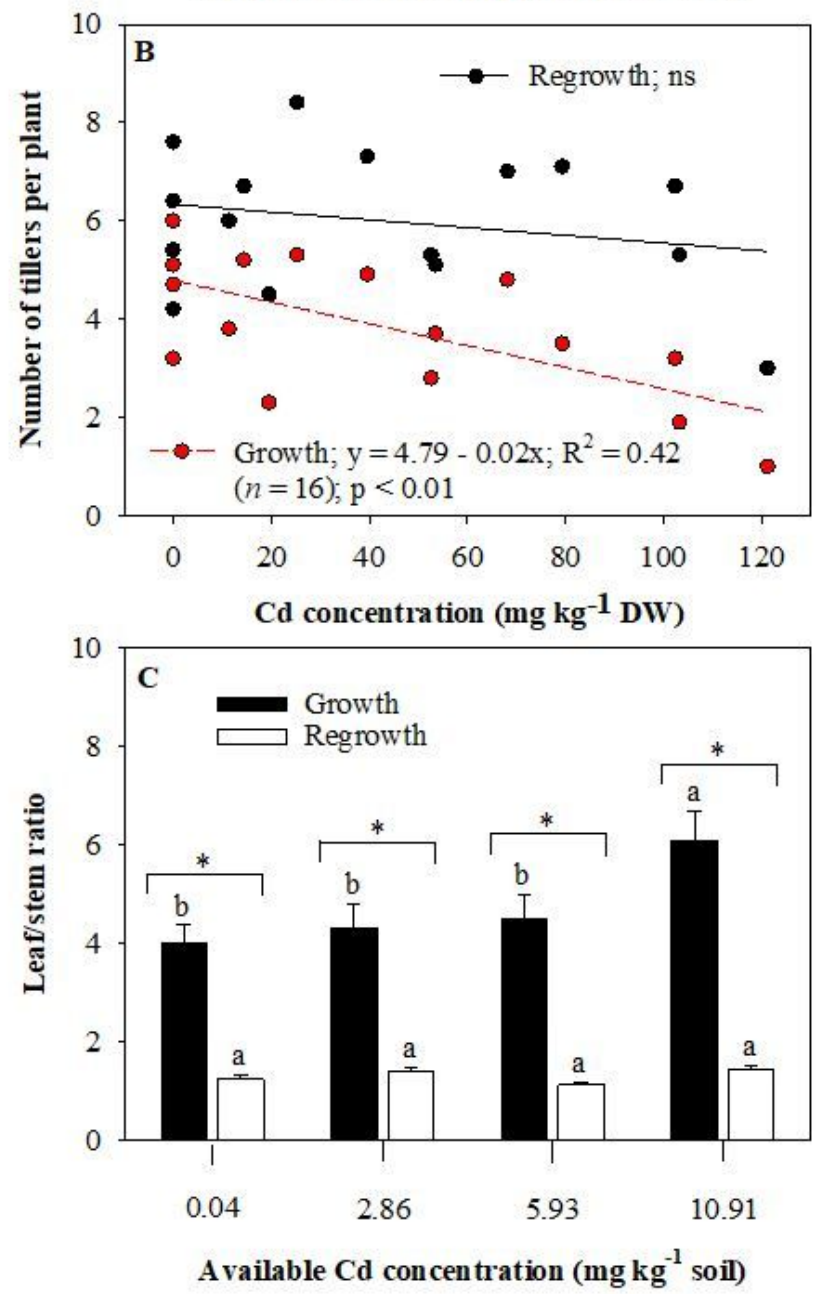

\section{Figure 3}

Number of tillers per plant (A), correlation between number of tillers per plant and $\mathrm{Cd}$ concentration in the basal node (B) and leaf/stem ratio (C) during the growth and regrowth of Panicum maximum established in non-polluted and $\mathrm{Cd}$-polluted Oxisol. Distinct letters on the bars indicate difference between $\mathrm{Cd}$ concentrations for each growth period of $P$. maximum $(n=4$, Tukey test, $\mathrm{p}<0.05)$. Asterisks represent differences at $p<0.05$ between growth periods within each Cd concentration (ANOVA, t-test). ns $=$ not significant 


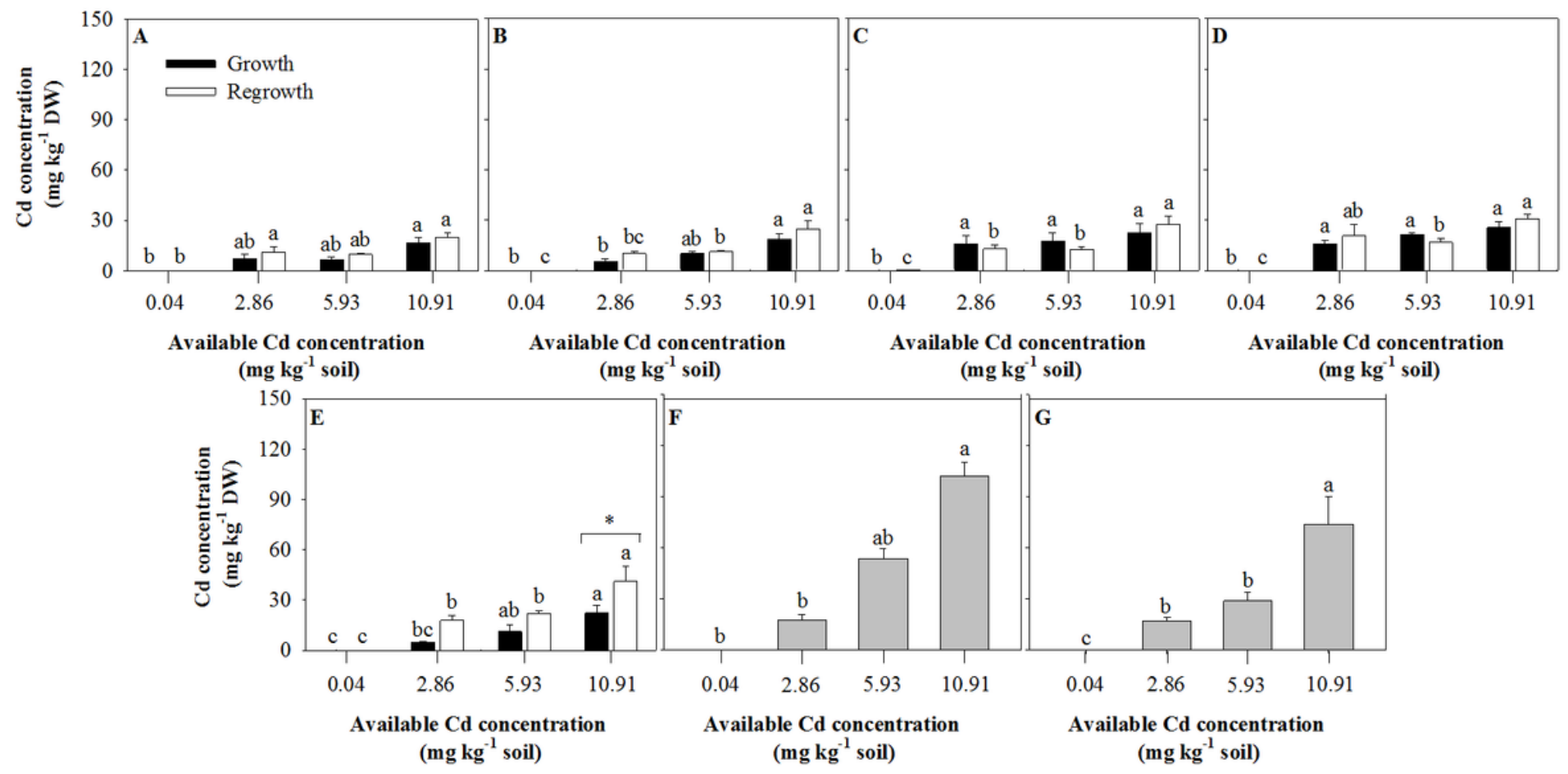

Figure 4

Cadmium concentration in the leaf I (A), leaf II (B), leaf III (C), other leaves (D), stems (E), basal node $(\mathbf{F})$ and roots $(\mathbf{G})$ of Panicum maximum established in non-polluted and Cd-polluted Oxisol. Distinct letters on the bars indicate difference between $C d$ concentrations for each growth period of $P$. maximum $(n=4$, Tukey test, $p<0.05)$. Asterisks represent differences at $p<0.05$ between growth periods within each $C d$ concentration (ANOVA, t-test) 

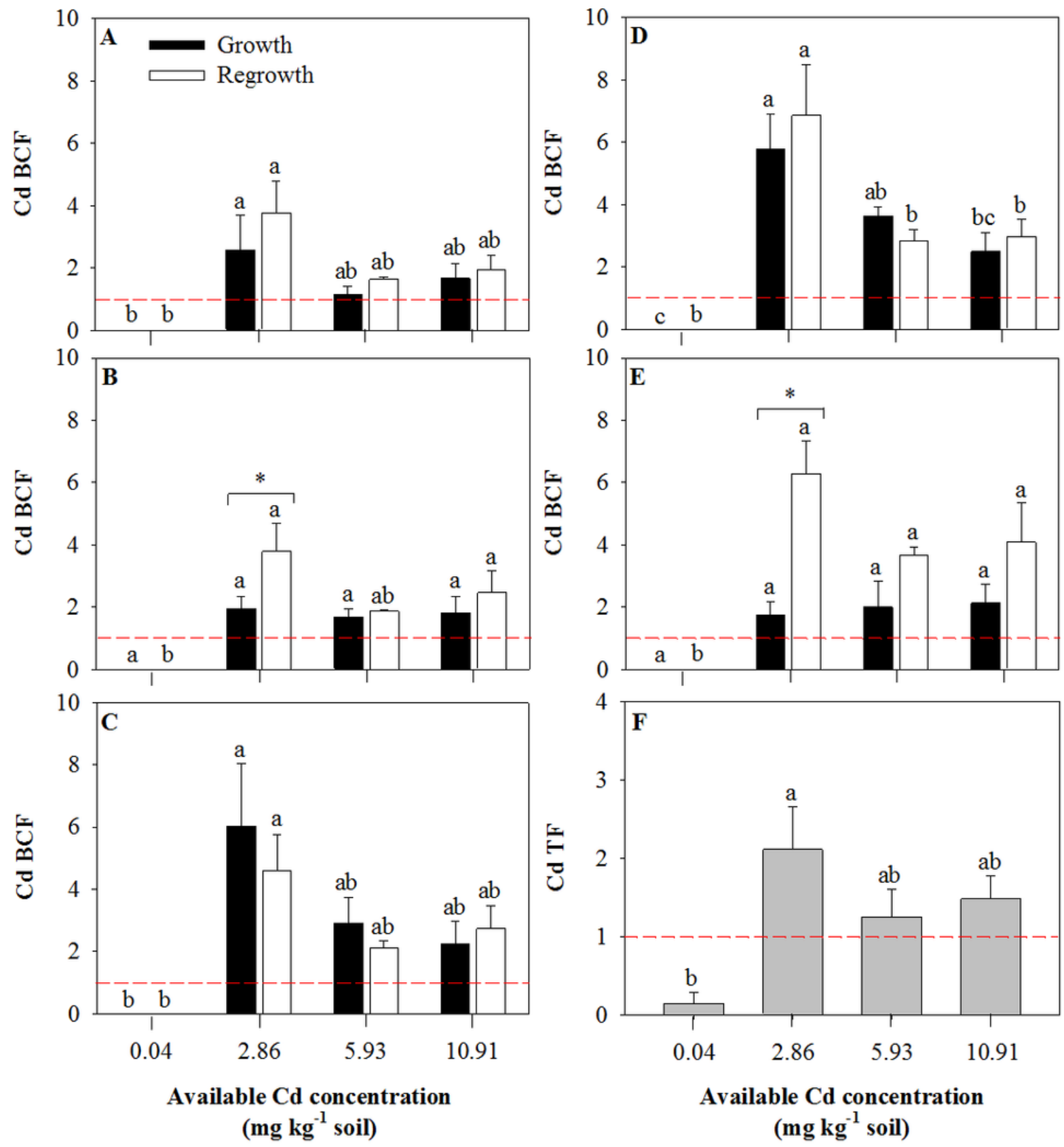

Figure 5

Bioconcentration factor - BCF in the leaf I (A), leaf II (B), leaf III (C), other leaves (D) and stems (E), and translocation factor - TF (F) of Panicum maximum established in non-polluted and Cd-polluted Oxisol. Distinct letters on the bars (A, B, C, D and E) indicate difference between Cd concentrations for each growth period of $P$. maximum $(n=4$, Tukey test, $p<0.05)$. Asterisks represent differences at $p<0.05$ between growth periods within each $\mathrm{Cd}$ concentration (ANOVA, t-test)

\section{Supplementary Files}

This is a list of supplementary files associated with this preprint. Click to download. 
- SupplementaryFigure1.docx

- SupplementaryFigure2.docx 